2017-12-13

Exploring foot and ankle impairments affecting mobility and balance in stroke (FAiMiS study)

\title{
Gorst, TR
}

http://hdl.handle.net/10026.1/10619

10.1016/j.physio.2017.11.026

Physiotherapy

Elsevier

All content in PEARL is protected by copyright law. Author manuscripts are made available in accordance with publisher policies. Please cite only the published version using the details provided on the item record or document. In the absence of an open licence (e.g. Creative Commons), permissions for further reuse of content should be sought from the publisher or author. 
included within the 'Safeguarding children and young people in music education' module of the Trinity Guildhall Exam Board Level 4 'Certificate for Music Educators'.

Conclusion(s): This project has had mixed success: within the school, against a background of an entrenched culture that broadly accepts pain as being a 'normal' part of playing a musical instrument, there is much work still to be done. Beyond the school, there is significant engagement at National level both from the Musicians Union and a National Exam Board.

Implications: The reflexive nature of Action research enabled the implementation of two previously unplanned strategies which have raised the profile of the issue of injury prevention in young musicians beyond the scope of the original project.

Funding acknowledgements: This EdD has been part funded by The Purcell School for Young Musicians, an award from the CSPs Physiotherapy Research Foundation, the Private Physiotherapy Education Fund, and Wolfson College, Cambridge.

https://doi.org/10.1016/j.physio.2017.11.025

\section{P025}

\section{Exploring foot and ankle impairments affecting mobility and balance in stroke (FAiMiS study)}

M. Cramp ${ }^{1}$, A. Rogers ${ }^{2, *}$, T. Gorst ${ }^{3,4}$, J. Freeman $^{3}$, J. Paton ${ }^{3}$, S. Morrison ${ }^{5}$, J. Marsden $^{3}$

${ }^{1}$ University of West of England, Faculty of

Health and Applied Science, Bristol, United

Kingdom

${ }^{2}$ Keele University, Health and

Rehabilitation, Keele, United Kingdom

${ }^{3}$ Plymouth University, School of Health

Professions, Plymouth, United Kingdom

${ }^{4}$ Northern Devon NHS Healthcare Trust,

Neuro Rehabilitation Service, Devon, United

Kingdom

${ }^{5}$ University of Brighton, School of Health

Sciences, Eastbourne, United Kingdom

Purpose: Foot posture abnormalities have been reported in $30 \%$ of people with stroke and are suggested to occur more frequently in people who walk indoors only. Stroke survivors also report that foot and ankle impairments are problematic and impact on everyday function. This project aimed to compare foot and ankle sensori-motor function of people with stroke with controls and to examine foot and ankle impairments as predictors of mobility and balance outcomes.

Methods: This cross-sectional observational study involved 180 people with stroke, >3 months post onset and mobilising independently, recruited from NHS stroke services and support groups at two UK sites, and 46 age- and gender-matched controls. Foot and ankle impairments assessed included foot posture, muscle strength, ankle and hallux joint dorsiflexion ROM, spasticity, sensation, foot pain, and dynamic foot loading. Mobility assessments included fast paced timed $10 \mathrm{~m}$ walk, and the forward functional reach was used as an indicator of balance. Descriptive statistics and data distribution was examined to determine the use of appropriate parametric and nonparametric analysis using SPSS version 22.0. Key variables were examined for co-variance and included in a multiple regression analysis.

Results: Foot and ankle impairment measures in stroke and control participants demonstrated significant differences between both groups in several variables including pain, spasticity, sensory loss and strength on the paretic side $(P<0.05)$. There were no significant differences in non-paretic muscle strength or in ankle and hallux dorsiflexion range. Multiple regression analysis on complete cases $(n=112 ; 12$ variables) identified that walking velocity could be predicted by strength, range of motion, peak pressure and sway $\left(R^{2}=0.55\right)$. Pooled analysis with imputed data found peak foot pressure of the paretic limb was an additional a significant predictor. Balance, assessed using the Forward Functional Reach Test, produced a multiple regression analysis of $R^{2}$ of 0.42 , with increased strength, range of motion, paretic foot peak pressure and reduced sway associated with greater forward reach. Pooled analysis with imputed data also indicated that peak foot pressure of the paretic limb was a significant predictor in the regression model. Whilst $18 \%$ of stroke participants reported a history of falls logistic regression analysis did not identify significant predictors of a falls history.

Conclusion(s): This work focused on foot and ankle impairments and as expected, found significant differences in key impairments between stroke and control participants. Notably, it has found that impairments such as strength and reduced ROM accompanied by measures of dynamic foot loading can, to some extent, predict overall mobility and balance outcomes following a stroke.

Implications: The findings indicate that foot interaction with the ground is an influential factor for mobility and balance and should be given due consideration in clinical practice. Foot and ankle muscle strength and ankle dorsiflexion joint range were key predictors of walking velocity and forward reach. Measures of dynamic foot loading, namely sway velocity and peak foot pressure for the paretic limb, were also consistent predictors. These impairments can be considered target areas for rehabilitation.

Funding acknowledgements: This work was funded by Dr William M. Scholl Podiatric Research \& Development Fund.

https://doi.org/10.1016/j.physio.2017.11.026 